\title{
Index
}

Numbers in italic type indicate figures

Acetabular fracture 212

Adenocarcinoma

colonic 81

laser surgery 224

gastric 60

intestinal 61

nasal 124-5

Aiming device for arthroscopy 193

Allergic rhinitis 122

Anaesthesia

for arthroscopy 194

for bronchoscopy 97-8

for colonoscopy 76

for endosurgery 97-8

for laparoscopy 159

for respiratory tract endoscopy 86-7

for rhinoscopy 98,114

for thoracoscopy $98,177-8$

for upper GI endoscopy 48

Analgesia for arthroscopy 194

Antrum 50-1

Arthroscopes 19, 189-90

for the carpus 209

for the elbow 202

for the hip 210

sheaths 20

for the shoulder 195

for the stifle 213

for the tarsus 217

Arthroscopy

anaesthetic considerations 194

analgesia 194

carpus $209-10$

elbow 202-3

hip 210-12

indications 188

instrumentation 24, 26-7, 189-93

patient preparation/positioning 193-4

postoperative care 195

preoperative work-up 188-9

shoulder 195-8

stifle 212-15

tarsus 217-18

Aspergillosis 121

topical therapy 127-30

Aspiration/lavage catheter 85
Bacteraemia after GI endoscopy 71-2

Bacterial rhinitis/sinusitis 122

Balloon dilation, oesophageal stricture 64

Benign mucosal antral polyp 59

Benign nasal polyp 127

Biceps rupture 201-2

Bicipital tenosynovitis 201

Biopsy

in arthroscopy 218

in bronchoscopy 92

in colonoscopy 75

in duodenoscopy 53-4

in gastroscopy 52

in flexible endoscopy 39-41

forceps $16,21,40,75$

intestinal 43

laparoscopic 162-5

in oesophagoscopy 49

in otoendoscopy 137

in rhinoscopy 116-17, 119-20

in thoracoscopy $179-80$

in urethrocystoscopy 146

Bipartite biceps tendon 197

Bougienage, oesophageal stricture dilation 64

Brachycephalic airway obstruction syndrome (BOAS) 94

Bradycardia after GI endoscopy 71

Breeding time assessment in the bitch 154-6

Bronchoalveolar lavage 91

Bronchoscope controls/handling 35

Bronchoscopy/Tracheobronchoscopy flexible

\section{biopsy 92}

complications 95-6

contraindications 85

indications 84

instrumentation 85-6

normal findings $88,89,90$

postoperative care 95

procedure 87-90

rigid

anaesthesia/sedation 97-8

Brush cytology

bronchial 91, 92

equipment 16,85

flexible endoscopy 39
Cameras 24

Cannulae 19, 20-1 for arthroscopy 191, 213

for laparoscopy 161 for thoracoscopy 176

Carbon dioxide lasers 221

Carcinoma, gastric 60

Cardia 51

Carpal chip fracture 210

Carpal synovitis 210

Carts 27, 30

Cervical abnormalities 154

Cervical catheterization 156

Cholecystectomy 173

Cholesteatoma 139

Chondrosarcoma, nasal 125-6

Client education 7

Coagulating electrode 16

Coccidioidomycosis 95

Colitis 79-80

Colonic neoplasia 81 laser surgery 224

Colonic vascular ectasia 82

Colonoscope, use in oesophagus 54-5

Colonoscopy

anaesthesia/sedation 76

biopsy 75

indications 73-4

instrumentation $74-5$

normal findings $77-8$

patient positioning/preparation

$$
\text { 76-7 }
$$

procedure 77-9

Constipation 82

Cruciate ligament injury 215-16

Cryptorchidism 170-1

Cystitis 152

Cystoscopes 18, 19

Cystoscopy

laparoscopic 171-3

transabdominal 147

(see also Urethrocystoscopy)

Cystostomy tube placement 147

Cystourethroscopes 145

Cytology brush 16, 85

Diaphragmatic hernia 182

Digital imaging 25-6

Diode laser systems 221-2

Disinfection of instruments 29

Dislodger 16

Duodenal biopsy 53-4 
Duodenal foreign bodies, removal 63

Duodenoscopy

indications 46

normal findings $56-7$

procedure 52-4

Duodenostomy tube placement 166

Ear

abnormalities 136-9

cleaning/flushing 133-5 complications 140

normal appearance 135

Electrosurgical equipment 26

Empyema, tympanic bulla 139

Endometrial disease 154

Endoscopes

care/cleaning 28, 32

disinfection 29

flexible 11

for colonoscopy 74-5

comparison with rigid 9

controls 34-6

for GI endoscopy 46-7

handling $32,33-4$

instruments $15-16$

for rhinoscopy 114, 117

selection 14-15

structure 12-13

for tracheobronchoscopy 85 , 86

for urethrocystoscopy 144 , 145

video versus fibreoptic 13-14

history 1-5

hybrid 86

light sources 21-3

rigid

for colonoscopy 75

comparison with flexible 9

for endosurgery 99-100

handling 100

instruments 19-21

for laparoscopy $99-100$

for proctoscopy 75

for rhinoscopy 117

selection 18-19

structure 17-18

for thoracoscopy $99-100$, 175-6

for urethrocystoscopy 145

for vaginoscopy 147

viewing angles 17

semi-rigid 18

sterilization 29

storage 29-30

(see also specific scopes)

Endoscopy, basic technique flexible

handling/care of equipment 33-4, 37-8

health and safety 31

patient considerations $31-2$

preparation 33

record keeping 41

sampling 39-41

rigid

health and safety 97 insufflation 98-9

patient preparation/ positioning 98

(see also specific procedures)

Endosurgery

anaesthetic considerations $97-8$

closure 107

future developments $9-10$

haemostasis 102

health and safety 97

instruments 99-100

insufflation $98-9$

irrigation 102

knot tying

extracorporeal 103-104

intracorporeal 104-107

laser 223-7

patient positioning 100

port placement 100-1

postoperative care $107-8$

procedure 101-2

sampling 103

suction 102

tissue dissection 102

(see also specific procedures)

Enemas 76, 82

Enteritis 61

Enteroscopes 47

Eosinophilic colitis 80

Eosinophilic enteritis 61

Ergonomics 28, 36

'False middle ear' 138

Feeding tubes see Duodenostomy, Gastrostomy, Jejunostomy

Fibrescopes

comparison with videoendoscopes 13-14 history 4

Fibrosing pleuritis 181

Fine-needle aspiration, flexible endoscopy 39

Flexible endoscopes see Endoscopes and specific scopes

Fluid management systems for arthroscopy 193

Forceps

biopsy $16,21,75$

grasping 16, 21, 191, 192

Foreign bodies

duodenal 63

gastric 62

nasal 122-3

nasopharyngeal 121

oesophageal 58,59

otic 137

removal

nasopharyngeal 117

tracheobronchial 95

Fractures upper GI tract $62-4$

acetabular 212

carpal 210

humeral 209

Fragmented medial coronoid process (FCP) 204-6

Friction brakes 36

Frontal sinus exploration 120

Fundus 51
Gastric biopsy 52

Gastric dilatation, after gastroscopy 71

Gastric foreign bodies 62 removal 63

Gastric neoplasia 59,60

Gastric over-distension 71

Gastric ulcers/erosions 59, 60

Gastritis 59, 60

Gastrointestinal perforation 71

Gastrointestinal tract

laser surgery 224

lower

biopsy 75

indications for endoscopy 73-4

instrumentation 74-5

patient preparation/ positioning 76-7 procedure $77-9$

upper

complications $71-2$

contraindications to endoscopy 46

indications for endoscopy 42-6

instrumentation 46-7

patient preparation/ positioning 47-8 procedure $48-54$

(see also specific conditions and procedures)

Gastropexy 167

Gastroscopes 3, 12, 13, 34-6, 47

Gastroscopy

indications 45

normal findings 56

procedure 49-52

theatre set up 28

Gastrostomy tube placement $65-70$, 166

Granulomatous colitis 80

Haemorrhage

after biopsy 173

in Gl endoscopy 39,71

in rhinoscopy 119,130

Haemostasis in endosurgery 102 , 168

Half hitch knot 107

Halogen light sources 22

Hand drill, arthroscopy 191, 192

Hasson technique 161

Health and safety

ear flushing 133

endosurgery 97

flexible endoscopy 31

lasers 223

Hiatal hernia 58

Hip dysplasia 212

Hip pain 212

Histiocytic colitis 80

Hypertrophic pylorogastropathy 59 , 60

Hysteroscopy 149

Ileocaecocolic junction 78

lleoscopy, indications 46 
Incomplete ossification caudal glenoid 199 humeral condyle 208-9

Inflammatory bowel disease 60,61 , 79-80

Instrument channels 12

Instrumentation

for arthroscopy 189-93

elbow 202

hip 210

shoulder 195

stifle 213

tarsus 217

for flexible endoscopy 15-16

for foreign body removal $62-3$

for haemostasis 102

for laparoscopy 158-9

for laser endosurgery 221-2

for otoendoscopy 131-2

for rhinoscopy 114

anterior 117

caudal 115

for rigid endoscopy 19-21

for thoracoscopy 175-7

for tracheobronchoscopy 85-6

(see also Endoscopes and other specific instruments)

Insufflation

complications 99

equipment 23

in GI tract flexible endoscopy 12 , 37,48

technique 98-9

Hasson 161

Veress needle 160-1

in vaginoscopy 148

Intestinal adenocarcinoma 61

Intestinal biopsy 43, 165-6

Intussusception

gastro-oesophageal 58

ileocolic/ileocaecal 82

Irrigation 12

in laparoscopy 102

in otoendoscopy 132,134

Irritable bowel syndrome 81

Jejunoscopy

indications 46

procedure 54

Jejunostomy tube placement $70-1$, 166

Knot tying

extracorporeal 103-4

intracorporeal 104-7

Laparoscopes 18, 19

Laparoscopy

anaesthetic considerations 159

biopsy

intestinal 165-6

liver 162-4

pancreatic 164

renal 164-5

closure 107

complications 173-4

endoscope choice 99-100 history $3,4-5$

instrumentation 158-9

insufflation 99

Hasson technique 161

Veress needle technique 160-1

laser surgery 225

port placement 100-1, 161-2

for intestinal biopsy 165

for liver biopsy 163

postoperative care 107-8

procedure 101-2, 162

suction/irrigation 102

surgical approaches 159-60

theatre set up 28

(see also specific surgical procedures)

Laryngeal paralysis 88

Lasers

carbon dioxide 221

diode 221-2

health and safety 223

lithotripsy 225-7

mass resection 126, 223-4

principles 220

selection 223

in urethrocystoscopy 147

Lateral glenohumeral ligament tears 200-1

Laxatives prior to colonoscopy 76

Leakage testing 13

Leiomyoma, oesophageal 58

Ligamentum arteriosum division 184-5

Light sources 21, 22-3

Lithotripsy, laser endosurgery 225-7

Liver biopsy 162-4

Lung lobe torsion 181-2

Lymphangiectasia $60,61,62$

Lymphocytic-plasmacytic colitis 79-80

Lymphoid follicles, reproductive tract 153

Lymphoid hyperplasia 116, 153

Lymphoma

colonic 81

gastric 60

nasal 124

Lymphoplasmacytic enteritis 61

Lymphosarcoma

intestinal 61

mediastinal 182

nasal 121

Masses

laser surgery $223-5$

otic 136-7

(see also Neoplasia)

Medial meniscus injury 215-16

Medial shoulder instability 199-200

Mediastinal biopsy 180

Mediastinal debridement 182

Megaoesophagus 57, 58

Melanoma, nasal 125

Monitors 24, 25

Myringotomy 138-9

complications 140
Nasal masses 123-7

laser resection 223-4

Nasopharyngeal polyps $126-7$

Nasopharyngeal stenosis 121-2

Neoplasia

colonic 81

duodenal 60,61

gastric 59,60

nasal 123-27

oesophageal 59

otic 136-7

pulmonary 181

urethral 136

vaginal 154

(see also specific tumours)

Oesophageal diverticulum 58

Oesophageal foreign bodies 59 removal 62

Oesophageal neoplasia 57,59

Oesophageal stricture $57,58,59,64$ dilation $64-5$

Oesophagitis 57, 58, 63

Oesophagoscopy

indications 45

normal findings $55-6$

procedure

flexible 49

rigid $54-5$

Ollulanus triscuspis 59,60

One-lung ventilation 178

Oslerus osleri 94

Osteoarthritis of the elbow 208

Osteochondritis dissecans elbow 206-7

shoulder 198-9

stifle 216

tarsus 218

Osteosarcoma, nasal 126

Otic biopsy 137

Otic discharge 133, 137

Otic foreign bodies 137

Otic masses 136-7

Otitis externa 133, 136, 138, 140

Otitis media 132, 133, 135, 137 . 138,140

Otoendoscopy

complications 140

indications 131

instruments 131-2

normal findings 135

patient preparation 132

postoperative care 140

preoperative work-up 132

procedure 132-135

sampling 133, 134

Otoscopes 18, 23, 131-2

Ovarian remnant removal 170

Ovariectomy 169-70

Ovariohysterectomy

laparoscopic 167-9

patient positioning 98

Pancreatic biopsy 164

Paramesonephric septum 153

Parasites

gastric 59,60

intestinal 60, 61, 62

(see also individual species) 
Patent ductus arteriosus (PDA), occlusion 185

Patient assessment/stabilization 8-9, 31

Patient preparation/restraint/ positioning 33 for arthroscopy 193-4 carpus 209 elbow 202-3 hip 210 shoulder 195 stifle 213 tarsus 217 for endosurgery 98 for Gl endoscopy 47-8, 76-7 for otoendoscopy 132 for respiratory tract endoscopy 87

for rhinoscopy anterior 117-18 caudal 114-15 for thoracoscopy 177 for urethrocystoscopy 145 for vaginoscopy 147-8

PEG tubes 66, 67 contraindications 66 indications 66 placement $67-9$ problems 70 removal 70 use $69-70$

Pericardectomy 182-3

Pericardial disease 181

Pericardial fenestration 186

Pericardiocentesis 182

Peristalsis 50

Persistent hymen 153

Peyer's patches 57

Physaloptera 59, 60

Pleural biopsy 179-80

Pleural effusion 181

Pneumolobectomy complete 184 partial 183-4

Pneumoperitoneum 98

Pneumothorax spontaneous 181 for thoracoscopy 98,179 Polyps

auropharyngeal 121 benign mucosal antral 59 bladder 172 nasopharyngeal 126-7 otic 136,137 rectal adenomatous $82-3$ urinary tract, laser resection 224-5

vaginal 154

Port placement arthroscopy carpus 209 elbow 203 hip 210-11 shoulder 195-6 stifle 213-14 tarsus $217-18$ laparoscopy 100-1, 161-2 intestinal biopsy 165 liver biopsy 163 ovariohysterectomy 167

pericardectomy 182

thoracic duct ligation 185

thoracoscopy 100-1, 179

Postoperative care

after arthroscopy 195

after bronchoscopy 95

after laparoscopy 107-8

after oesophageal stricture dilation 64-5

after otoendoscopy 140

after rhinoscopy 130

after thermal capsulorrhaphy 200

after thoracoscopy 186

after urethrocystoscopy 157

Power shavers 26-7, 192-3

Premedication/sedation

for bronchoscopy 97

for colonoscopy 76

for respiratory tract endoscopy 86

for rhinoscopy 114

for upper Gl endoscopy 48

Pre-oxygenation for respiratory tract endoscopy 86,98

Pressure valve 13

Probes, arthroscopic 191

Proctoscopy

indications 73

procedure 79

Prostatic disease 151

Pseudomonas aeruginosa 134, 136

Pulmonary biopsy 180

Pulmonary oedema 95

Pumps 23 for arthroscopy 24

Pyloric intubation 53

Pyloric stenosis 59

Rectal adenomatous polyps 82-3

Rectal stricture 83

Red-out 37

Remnant hymen 153

Renal biopsy 164-5

Respiratory tract

anaesthesia 86-7

indications for endoscopy 84

instrumentation 85-6

patient preparation/positioning 87

premedication 86

(see also specific conditions and

Rhinitis procedures)

allergic 122

bacterial 122

chronic 121

foreign body 122-3

fungal, topical therapy $127-30$

Rhinoscope 18

Rhinoscopy

anaesthesia 98

anatomical considerations 109-10

anterior

biopsy 119-20

instrumentation 117

normal findings 118-19 patient preparation/

positioning 117-18

caudal

procedure 118-19

biopsy 116--17

foreign body removal 117

instrumentation 114

normal findings 116

patient preparation/ positioning 114-15

procedure 115-16

rigid 117

complications 130

indications 110-12

intraoperative work-up 112-13

laser surgery 223-4

postoperative care 130

preoperative work-up 112

Rigid endoscopes see Endoscopes and specific scopes

Rod lens system 17

Roeder knot 104, 105

Scissors 16

Sedation see Premedication/sedation

Septic arthritis elbow 209

hip 212

Shavers 26-7, 192-3

Sheaths 19, 20

Snares 16

Specimen retrieval bags 103

Spirocerca lupi 57, 59

Squamous cell carcinoma, oesophageal 58

Sterilization 29

Suction 12

artefact 35

in laparoscopy 102

in otoendoscopy 132,134

Synovial biopsy, tarsal 218

T-adaptor, swivel tip 86

Teamwork 27

Telescopes 17

Theatre set up 7,27-8, 189

Thermal capsulorrhaphy 199-200

Thoracic duct occlusion 185-6

Thoracoscopy

anaesthesia $98,177-8$

biopsy 179-80

closure 107

complications 186

indications 175

instrumentation 99-100, 175-7

laser surgery 225

normal findings 180-1

patient preparation/positioning 98,177

port placement 100-1, 179

postoperative care 186

preoperative work-up 177

procedure $102,178-80$

Tonsillar inflammation 113

Toxocara 61

Tracheal collapse 93-4

Tracheal mass 94

Tracheal stent 94 


\section{Index}

Tracheobronchitis 92

Tracheobronchoscopy see Bronchoscopy/ Tracheobronchoscopy

Training 8, 31

Transabdominal cystoscopy 147

Transbronchial aspiration/biopsy needles 86

Transitional cell carcinoma

bladder 153

urethral 147, 151

laser surgery 224

Transmissible venereal tumour (TVT) 154

Transurethral laser lithotripsy 225-7

Trichuris vulpis 78, 82

Trocars 20

Tympanic membrane

absence 137

displacement 138

normal findings 135

rupture 138

Typhlitis 82

Ulcerative rhinitis 119

Ulcers

ear canal 136

gastric 59

Ultrasonic broncho-fibre-videoscope 86

Uncinaria 62

Ununited anconeal process 207-8
Ureteral ectopia 147, 151-2

Ureteroceles 152

Urethral dys-synergia 151

Urethral neoplasia 147, 151 laser surgery 224

Urethral obstruction 151

Urethral sphincter mechanism incompetence (USMI) 151

Urethrocystoscopes 148

Urethrocystoscopy/urethroscopy anatomical considerations 142-3 biopsy 146 complications 157

indications 143

instrumentation 144-5

intraoperative work-up 144

laser techniques 147, 224

normal findings 146

patient preparation/positioning

$$
98,145
$$

postoperative care 157

preoperative work-up 144

procedure 145-7

urolith removal 147

Urethroliths 226

Urethroscopy see Urethrocystoscopy

Urinary tract

infection (UTI) 152-3, 157

lower, laser surgery 224-5

(see also specific conditions and procedures)

Urocystoliths 225

Urolithiasis 153, 225
Uroliths

laser lithotripsy 225-7

removal 147

Vaginal hyperplasia 154

Vaginal neoplasia 154

Vaginal polyps 154

Vaginoscopy

breeding time assessment in the bitch 154-6

complications 157

endoscopes 147

normal findings

non-oestrous bitch 149

oestrous changes $149-50$, 155

patient preparation/positioning 147-8

procedure 148-9

Veress needle 23

technique 160-1

Vestibulovaginal stenosis 153-4

Video-endoscopes

comparison with fibrescopes 13-14

controls 36

imaging systems 24-5

structure 13

Video-otoscopy see Otoendoscopy

Xenon light sources 22 